\title{
TRAPPING OF ELECTRON CLOUD IN ILC/CESRTA QUADRUPOLE AND SEXTUPOLE MAGNETS
}

\author{
L. Wang and M. Pivi, SLAC, Menlo Park, CA 94025, U.S.A.
}

\begin{abstract}
The Cornell Electron Storage Ring (CESR) has been reconfigured as an ultra low emittance damping ring for use as a test accelerator (CesrTA) for International Linear Collider (ILC) damping ring $R \& D$ [1]. One of the primary goals of the CesrTA program is to investigate the interaction of the electron cloud with low emittance positron beam to explore methods to suppress the electron cloud, develop suitable advanced instrumentation required for these experimental studies and benchmark predictions by simulation codes. This paper reports the simulation of the electron-cloud formation in CESRTA and ILC quadrupole and sextupole magnets using the 3D code CLOUDLAND. We found that electrons can be trapped with a long lifetime in a quadrupole and sextupole magnet due to the mirror field trapping mechanism. We study the effects of magnet strength, bunch current, antechamber effect, bunch spacing effect and secondary emission yield (SEY) in great detail.
\end{abstract}

\section{INTRODUCTION}

The development of an electron cloud in magnets is the main concern where a weak solenoid field is not effective. Quadrupole and sextupole magnets have mirror field configurations which may trap electrons by the mirror field trapping mechanism [2]. Fig.1 shows the orbit of a trapped electron in a quadrupole magnet. The electron makes gyration motion (called transverse motion) and also moves along the field line (called longitudinal motion). At the mirror point (middle of the field line), there is a maximum longitudinal energy and minimum transverse energy. When the electron moves away from the mirror point, its longitudinal energy reduces and the transverse energy increases as the magnetic field increases. If the magnetic field is strong enough, the longitudinal energy becomes zero at one point and then the electron is turned back by the strong field. Note that the electrons are trapped in the region near the middle of the field lines. Although all quadrupole and sextupole magnets can trap electrons in principle, the trapping mechanism is also greatly sensitive to the detail dynamics of the electrons [3]. Both the positron beam and the spacing charge force of electron cloud itself play important roles. This paper reports the simulation of electron cloud in CESRTA/ILC quadrupole and sextupole magnets. Table 1 shows the main parameters used in the simulation.

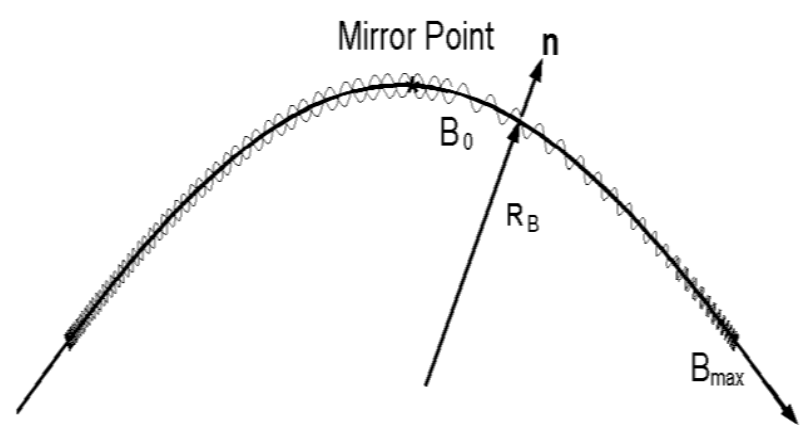

Fig.1: Trapping of an electron by a mirror field (quadrupole field here) from CLOUDLAND code

Table 1: Main simulation parameters for CESRTA and ILC positron damping ring

\begin{tabular}{|c||c||c|}
\hline Description & CESRTA & ILC \\
\hline \hline Beam energy $(\mathrm{GeV})$ & 5.289 & 5.0 \\
\hline Circumference $(\mathrm{m})$ & 768.43 & 3238 \\
\hline \hline Bunch length $(\mathrm{mm})$ & $15.0 / 17.24$ & 6.0 \\
\hline \hline Beam size $(\mathrm{mm})$ & $1.56 / 0.15$ & $0.27 / 0.005$ \\
\hline \hline Bunch spacing $(\mathrm{ns})$ & 14 & $3 / 6$ \\
\hline \hline Bunch number per train & 45 & 45 \\
\hline \hline Bunch intensity $\left(\times 10^{10}\right)$ & $0.75 \sim 1.6$ & 2.1 \\
\hline
\end{tabular}

\section{TRAPPING IN CESRTA QUADRUPOLE}

In principle, electron cloud can be trapped in a quadrupole magnet due to the mirror field trapping. However, certain conditions are required for a deep trapping [3]. Electron cloud in a quadrupole magnet is sensitive to other parameters besides secondary emission, bunch current and beam filling pattern. Fig. 2 shows the build-up of the electron cloud in a quadrupole magnet with a field gradient of $0.517 \mathrm{~T} / \mathrm{m}$. The beam has one bunch train consisting of 45 bunches followed by a long train gap of $1.93 \mu \mathrm{s}$. The electron cloud reaches saturation level after 10 turns $(25 \mu s)$. In contrast to the dipole magnet case, where electrons couldn't survive such long train gap, the electrons in quadrupole magnets surviving from the long train gap are trapped electrons. About 50\% electrons can survive from the long gap as shown in the figure. Fig. 3 shows the evolution of an electron cloud during the train gap. The $1^{\text {st }}$ picture in the figure is the electron cloud just after the passage of the last bunch along the bunch train. The positron bunches see an electron cloud like an Octupole field pattern in this case because the trapped electrons near the mirror field region are added to the quadrupole field pattern. After about 280 $n s$, only the electrons located at the minimum field region

\footnotetext{
*Work supported by the U.S. Department of Energy under contract DE-AC02-76SF00515 and DE-FC0208ER41538

\# email address: wanglf@slac.stanford.edu
} 
of the mirror field lines are survived, which shows excellent agreement with mirror field trapping mechanism.

FIG. 4 and 5 shows the build-up and evolution of electron cloud during the train gap with a field gradient of 9.2 T/m. Other parameters are the same as Fig. 2 and 3. Differently in this case, we can clearly see the quadrupole pattern: electrons moving along the magnetic field lines. The stronger magnetic field makes the trapped electrons closer to the beam, and therefore there is a larger electron density near the beam. As a result, these trapped electrons are more important for the beam dynamics. Note that there is a similar average electron density with the two field gradients.

FIG. 6 shows the bunch current effect with a field gradient of $1.0 \mathrm{~T} / \mathrm{m}$. There is a slow build-up for low bunch current. FIG.7 is the peak electron density for various beam current and magnetic field strength. It is interesting that the peak average electron density at the end of the bunch train is close to a linear function of the bunch current. On the other hand, the electron density near the beam is close to a cubic function of bunch current. There is a larger density for a stronger magnetic field. The electron density near the beam is more sensitive to the magnetic field strength. The strong field confines the electrons more close to the beam center.

In a quadrupole magnet, there is a large electron flux near the middle the magnet poles as shown in FIG. 3 and 5. Note that the trapped electron cloud in FIG. 3 doesn't contribute a large electron flux at the surface of the beam pipe because they are trapped inside the beam chamber. The simulated electron flux has large peak at the center of the magnets poles (not shown here). This qualitatively agrees with the observation in CESRTA [4]. The trapped electron cloud in quadrupole magnet also has been observed experimentally in PSR [5].

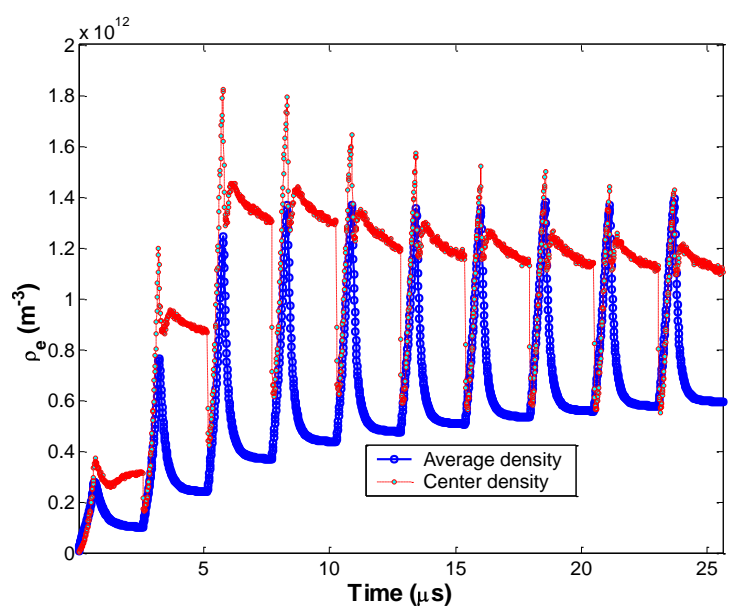

FIG. 2. Electron build-up in a quadrupole magnet with a field gradient of $0.517 \mathrm{~T} / \mathrm{m}$.
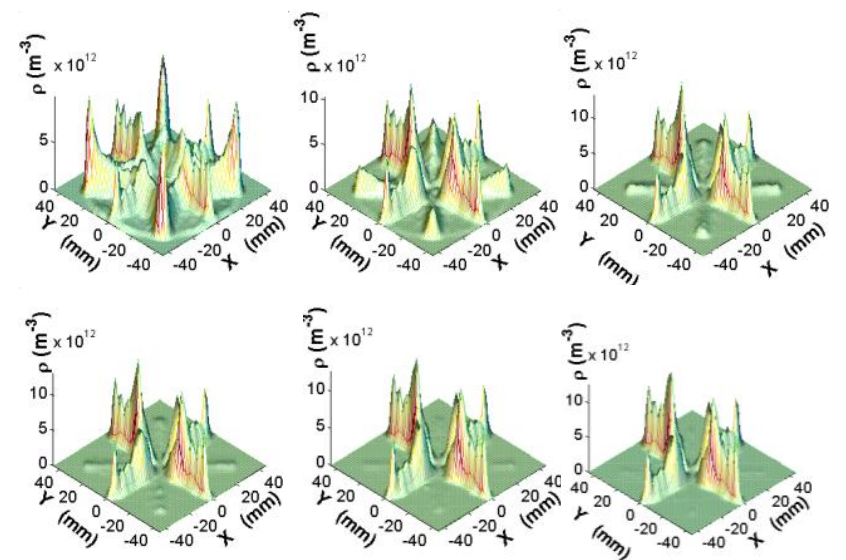

FIG. 3. Evolution of electron cloud during the train gap, frames separated by $\Delta \mathrm{t}=70 \mathrm{~ns}$. Parameters used for simulation: Bunch length $17.24 \mathrm{~mm}$, bunch current 1.0 $m A$, bunch spacing $14 n s$, field gradient $0.517 \mathrm{~T} / \mathrm{m}$, peak SEY 2.0, energy at peak SEY $310 \mathrm{eV}$, photon flux 0.21 photons $/ \mathrm{m} /$ particle, reflectivity $20 \%$.

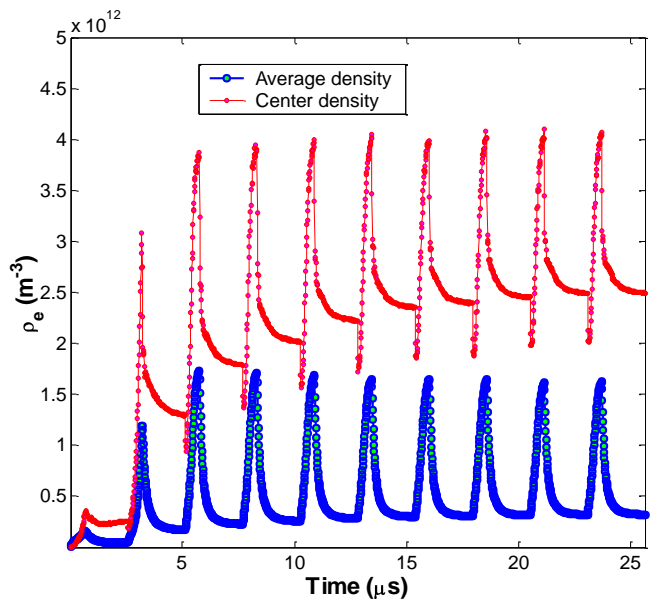

FIG. 4. Electron build-up in a quadrupole magnet with a field gradient of $9.2 \mathrm{~T} / \mathrm{m}$.
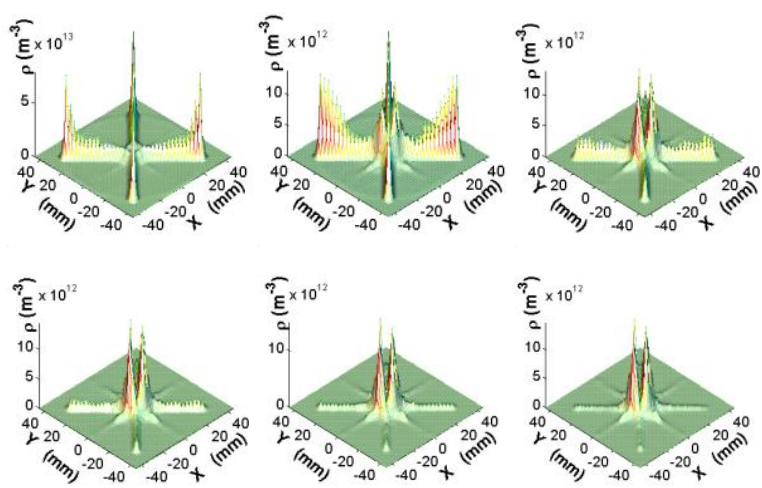

FIG. 5. Evolution of electron cloud during the train gap with a field gradient of $9.2 \mathrm{~T} / \mathrm{m}$. Other Parameters are the same as those in Fig. 3. Frames are separated by $\Delta \mathrm{t}=70$ $n s$. 


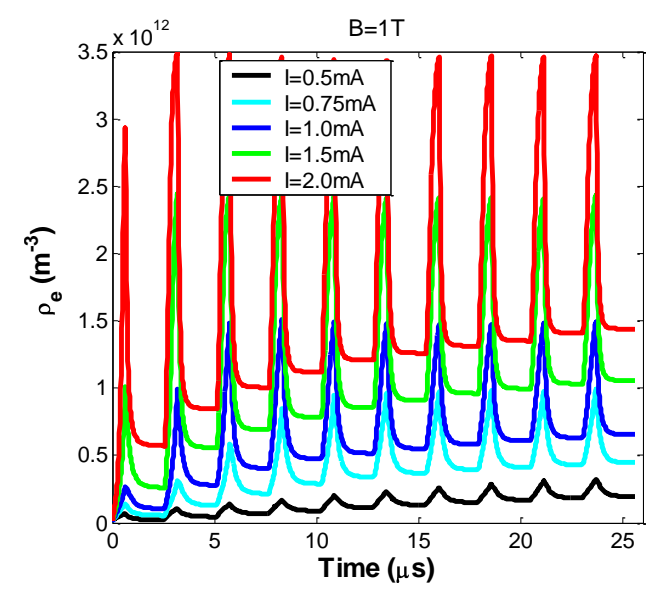

FIG. 6. Bunch current effect on electron build-up with a magnetic field of $1 \mathrm{~T}$.
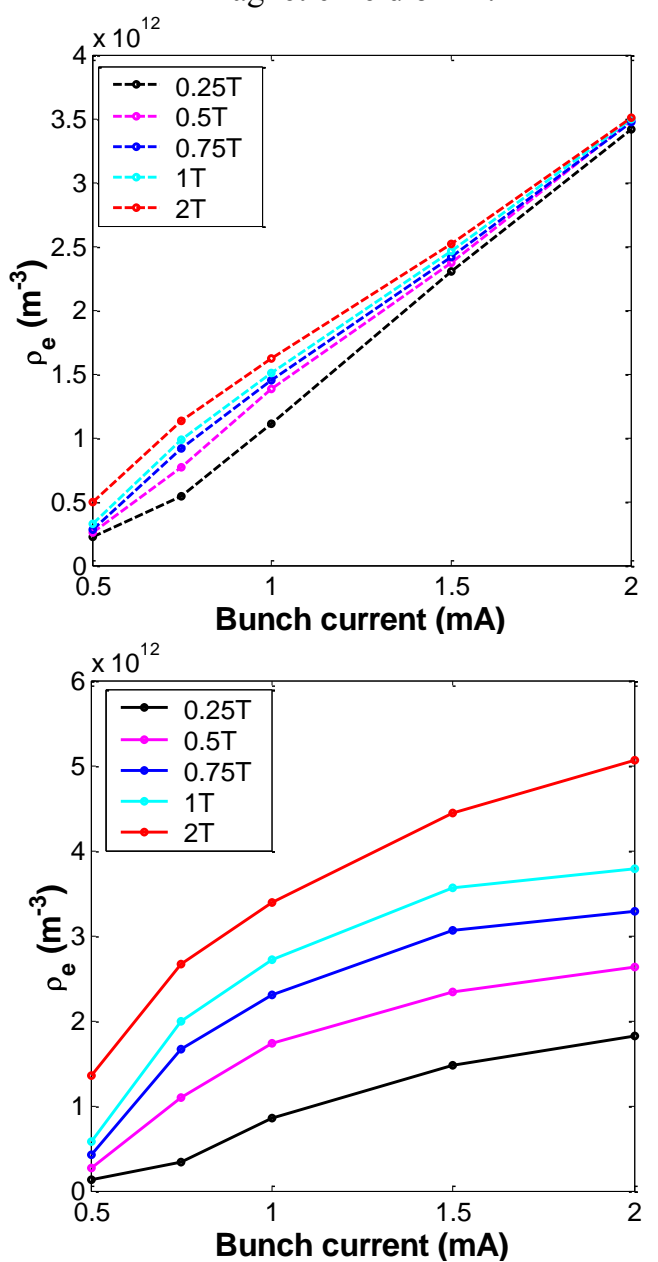

FIG. 7. Dependence of electron density on bunch current and magnetic field. Top: average electron density; bottom: electron density near the beam.

\section{TRAPPING OF ELECTRON CLOUD IN ILC QUADRUPOLE AND SEXTUPOLE}

Many configurations have been studied for ILC positron damping ring in order to optimize the electron cloud effect on the beam dynamics, for instance, the ring circumference, bunch spacing, ante-chamber effect, coating (SEY) effect. In this study, we only study $3 \mathrm{~km}$ ring. We systematically scan the following parameters: bunch spacing from $3 \mathrm{~ns}$ to $6 \mathrm{~ns}$, SEYs from 0.9 to 1.4 , and ante-chamber protection effect of $0 \%, 90 \%$ and $98 \%$, respectively. FIG. 8 shows the build-up of electron cloud in ILC quadrupole magnet without ante-chamber effect for different SEYs and bunch spacing. FIG. 9 shows the build-up of electron cloud with $98 \%$ ante-chamber reduction effect for different SEYs and bunch spacing. A larger number of electrons $(30 \% \sim 70 \%)$ can be trapped and survive from the long train gap and there is a slow build-up and slow decay comparing with electron in a dipole magnet. In general, it takes several bunch trains (even turns in CESRTA case) for the electron cloud to get saturation level. It saturates quickly without ante-chamber as expected due to the large photons flux.

The maximum electron densities along the bunch train with various configurations are listed in Table 2-5. Electron density in sextupole is lower than that in the quadrupole magnet. Here we discuss the electron in quadrupole magnet only. There is a larger (a factor 2.3 1.6) average electron density in 3ns spacing case comparing with $6 \mathrm{~ns}$ spacing. The electron density near the beam is also always smaller with a $6 \mathrm{~ns}$ bunch spacing beam.

The effect of SEY is significant with ante-chamber while it become less effective without ante-chamber because the strong space charge force limits the saturation density. In all cases, there is a larger density for a larger SEY. And there is a long saturation time for low SEYs.

The effect of ante-chamber is complicated by the space charge effect. Antechamber is effective in reduction of the electron cloud density when SEY is small $(<=1.0)$; On the other hand, its mitigation effect becomes smaller with a larger SEY because the secondaries are dominant. With ante-chamber, there is always a small average electron density. However when SEY is larger (>1.2) the density near the beam can be larger comparing without antechamber case. Simulation shows there is a different electron distribution with and without ante-chamber protection effect. Detail study finds the space charge effect without ante chamber play a very important role on the electron distribution. FIG. 10 shows the electron distribution with ante-chamber protection effect. There is a large density around the beam. However, there is a lower density near the beam without ante-chamber as shown in FIG.11. The space charge force reduces the density near the beam in this case. In a short summary, the ante chamber has to be coated to reduce its SEY in order to take its advantage of reduction of the photons. With a $\mathrm{SEY}>1.1$, the ante-chamber doesn't reduce the electron density near the beam! 


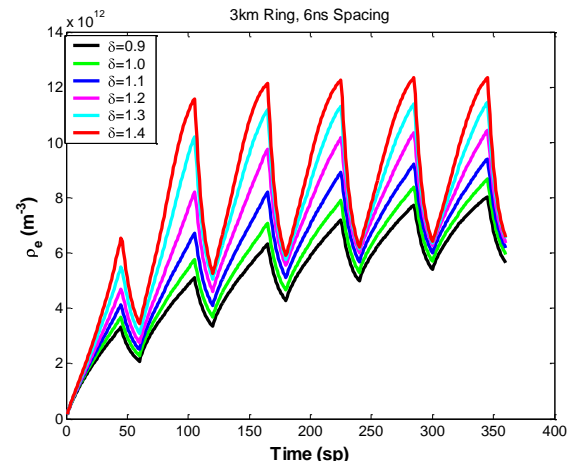

(a)

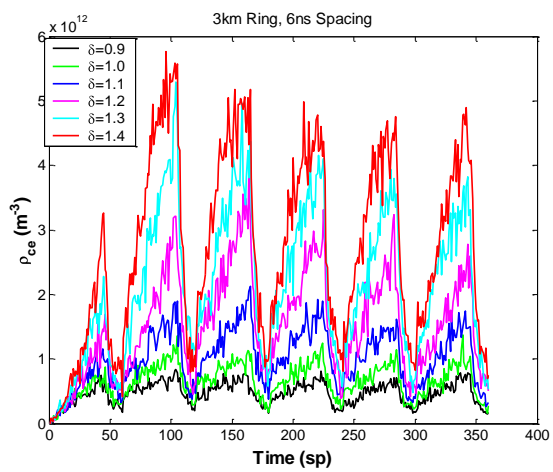

(b)

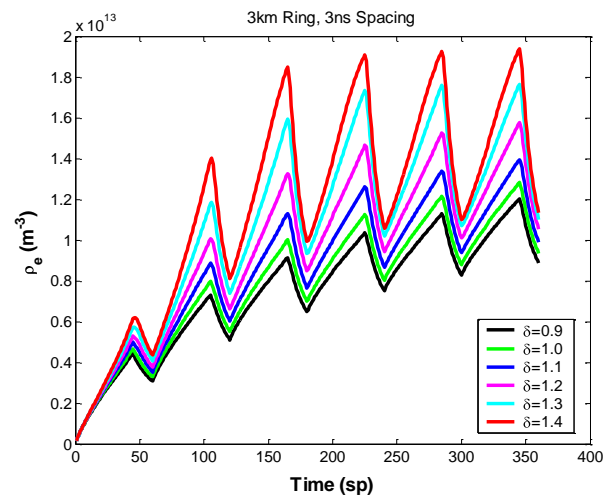

( C)

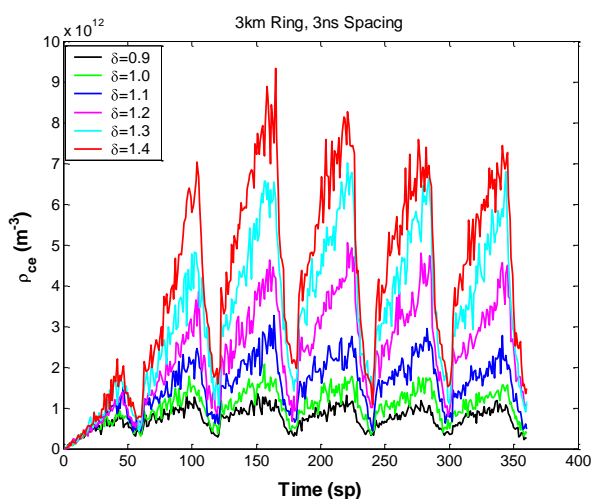

(d)

FIG. 8. Electron build-up in the ILC quadrupole magnet without ante-chamber protection. (a) 6ns spacing, average density; (b) 6ns spacing, electron density near the beam; (c) 3ns spacing, average density; (d) 3ns spacing, electron density near the beam

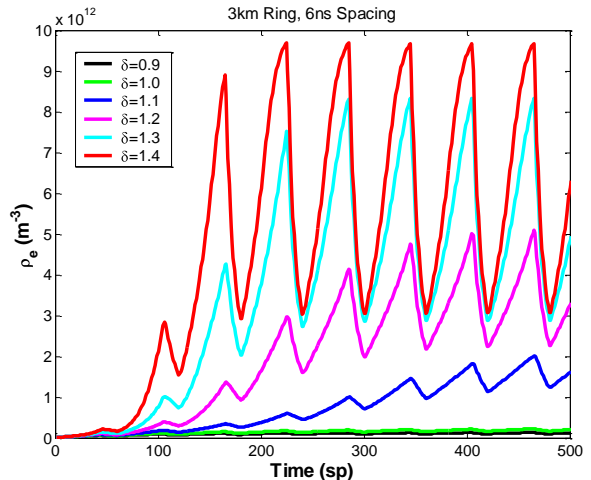

(a)

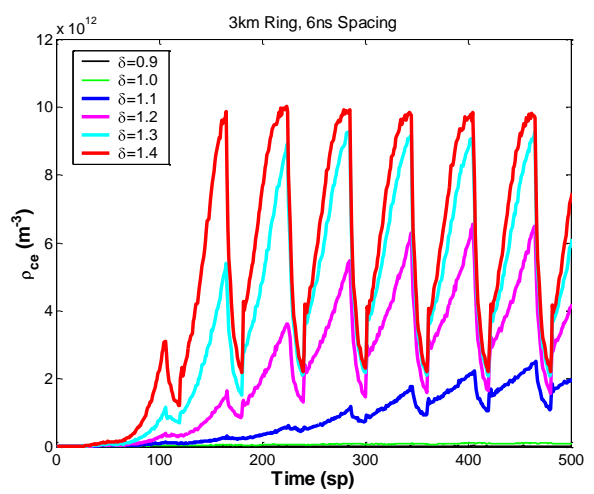

(b)

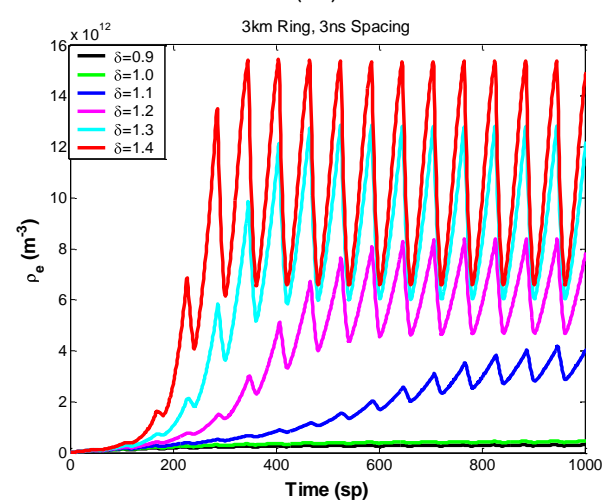

(C)

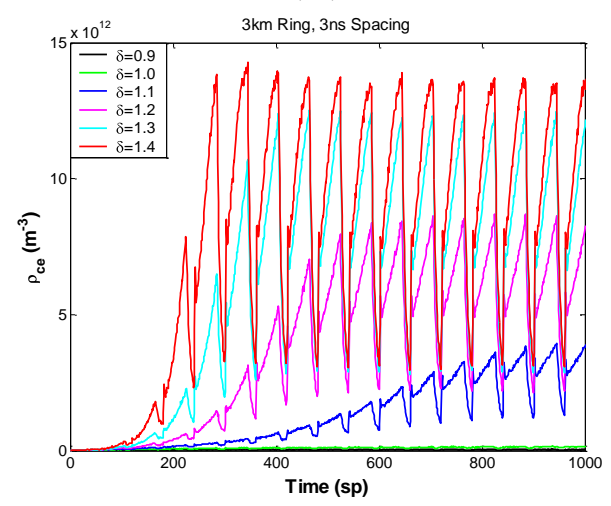

(d)

FIG. 9. Electron build-up in the ILC quadrupole magnet with $98 \%$ reduction of photons due to the ante-chamber. (a) 6ns spacing, average density; (b) 6ns spacing, electron density near the beam; (c) 3ns spacing, average density;

(d) 3ns spacing, electron density near the beam 
Table 2: Peak average density in ILC quadrupole magnet $\left(1 \times 10^{12} \mathrm{~m}^{-3}\right)$

\begin{tabular}{|c|c|c|c|c|c|c|}
\hline SEY & \multicolumn{2}{|c|}{$\begin{array}{c}\text { Antechamber } \\
\text { protection }=0 \%\end{array}$} & \multicolumn{2}{c|}{$\begin{array}{c}\text { Antechamber } \\
\text { protection }=90 \%\end{array}$} & \multicolumn{2}{c|}{$\begin{array}{c}\text { Antechamber } \\
\text { protection }=98 \%\end{array}$} \\
\hline & $\begin{array}{c}3 \mathrm{~ns} \\
\text { spacing }\end{array}$ & $\begin{array}{c}6 \mathrm{~ns} \\
\text { spacing }\end{array}$ & $\begin{array}{c}3 \mathrm{~ns} \\
\text { spacing }\end{array}$ & $\begin{array}{c}6 \mathrm{~ns} \\
\text { spacing }\end{array}$ & $\begin{array}{c}3 \mathrm{~ns} \\
\text { spacing }\end{array}$ & $\begin{array}{c}6 \mathrm{~ns} \\
\text { spacing }\end{array}$ \\
\hline 0.9 & 12.0 & 8.04 & 1.64 & 0.70 & 0.315 & 0.136 \\
\hline 1.0 & 12.8 & 8.70 & 2.20 & 1.0 & 0.466 & 0.260 \\
\hline 1.1 & 13.85 & 9.41 & 4.82 & 2.38 & 4.30 & 2.20 \\
\hline 1.2 & 15.74 & 10.44 & 8.70 & 5.25 & 8.40 & 5.13 \\
\hline 1.3 & 17.50 & 11.45 & 13.2 & 8.58 & 12.8 & 8.35 \\
\hline 1.4 & 19.20 & 12.35 & 15.8 & 9.97 & 15.37 & 9.7 \\
\hline
\end{tabular}

Table 3: Peak central density in ILC quadrupole magnet

\begin{tabular}{|c|c|c|c|c|c|c|}
\multicolumn{9}{c|}{$\left(1 \times 10^{12} \mathrm{~m}^{-3}\right)$} \\
\hline & \multicolumn{2}{|c|}{$\begin{array}{c}\text { Antechamber } \\
\text { protection }=0 \%\end{array}$} & \multicolumn{2}{|c|}{$\begin{array}{c}\text { Antechamber } \\
\text { protection }=90 \%\end{array}$} & \multicolumn{2}{|c|}{$\begin{array}{c}\text { Antechamber } \\
\text { protection }=98 \%\end{array}$} \\
\hline & $\begin{array}{c}3 \mathrm{~ns} \\
\text { spacing }\end{array}$ & $\begin{array}{c}6 \mathrm{~ns} \\
\text { spacing }\end{array}$ & $\begin{array}{c}3 \mathrm{~ns} \\
\text { spacing }\end{array}$ & $\begin{array}{c}6 \mathrm{~ns} \\
\text { spacing }\end{array}$ & $\begin{array}{c}3 \mathrm{~ns} \\
\text { spacing }\end{array}$ & $\begin{array}{c}6 \mathrm{~ns} \\
\text { spacing }\end{array}$ \\
\hline 0.9 & 1.0 & 0.67 & 0.18 & 0.11 & 0.035 & 0.020 \\
\hline 1.0 & 1.6 & 0.9 & 0.55 & 0.35 & 0.135 & 0.14 \\
\hline 1.1 & 2.2 & 1.5 & 3.25 & 2.2 & 4.0 & 2.75 \\
\hline 1.2 & 4.4 & 2.6 & 7.5 & 6.0 & 8.7 & 6.55 \\
\hline 1.3 & 6.7 & 3.8 & 11.5 & 8.5 & 12.4 & 9.2 \\
\hline 1.4 & 7.5 & 4.9 & 13.0 & 9.5 & 13.6 & 9.9 \\
\hline
\end{tabular}

Table 4: Peak average density in ILC sextupole magnet

\begin{tabular}{|c|c|c|c|c|c|c|}
\hline SEY & \multicolumn{2}{|c|}{$\begin{array}{c}\text { Antechamber } \\
\text { protection }=0 \%\end{array}$} & \multicolumn{2}{c|}{$\begin{array}{c}\text { Antechamber } \\
\text { protection }=90 \%\end{array}$} & \multicolumn{2}{|c|}{$\begin{array}{c}\text { Antechamber } \\
\text { protection }=98 \%\end{array}$} \\
\hline & $\begin{array}{c}3 \mathrm{~ns} \\
\text { spacing }\end{array}$ & $\begin{array}{c}6 \mathrm{~ns} \\
\text { spacing }\end{array}$ & $\begin{array}{c}3 \mathrm{~ns} \\
\text { spacing }\end{array}$ & $\begin{array}{c}6 \mathrm{~ns} \\
\text { spacing }\end{array}$ & $\begin{array}{c}3 \mathrm{~ns} \\
\text { spacing }\end{array}$ & $\begin{array}{c}6 \mathrm{~ns} \\
\text { spacing }\end{array}$ \\
\hline 0.9 & 11.3 & 6.4 & 1.07 & 0.52 & 0.2 & 0.10 \\
\hline 1.0 & $>11.8$ & 7.05 & 1.2 & 0.605 & 0.22 & 0.116 \\
\hline 1.1 & $>12.4$ & 7.75 & $>1.35$ & 0.73 & 0.248 & 0.135 \\
\hline 1.2 & $>13.2$ & $>8.46$ & $>1.55$ & 0.94 & 0.290 & 0.174 \\
\hline 1.3 & $>13.8$ & $>9.3$ & 1.95 & 1.56 & 0.354 & 0.303 \\
\hline 1.4 & 14.8 & 10.2 & 3.03 & 3.25 & 0.65 & $>2.0$ \\
\hline
\end{tabular}

Table 5: Peak central density in ILC sextupole magnet $\left(1 \times 10^{12} \mathrm{~m}^{-3}\right)$

\begin{tabular}{|c|c|c|c|c|c|c|}
\hline SEY & \multicolumn{2}{|c|}{$\begin{array}{c}\text { Antechamber } \\
\text { protection }=0 \%\end{array}$} & \multicolumn{2}{c|}{$\begin{array}{c}\text { Antechamber } \\
\text { protection }=90 \%\end{array}$} & \multicolumn{2}{c|}{$\begin{array}{c}\text { Antechamber } \\
\text { protection }=98 \%\end{array}$} \\
\hline & $\begin{array}{c}3 \mathrm{~ns} \\
\text { spacing }\end{array}$ & $\begin{array}{c}6 \mathrm{~ns} \\
\text { spacing }\end{array}$ & $\begin{array}{c}3 \mathrm{~ns} \\
\text { spacing }\end{array}$ & $\begin{array}{c}6 \mathrm{~ns} \\
\text { spacing }\end{array}$ & $\begin{array}{c}3 \mathrm{~ns} \\
\text { spacing }\end{array}$ & $\begin{array}{c}6 \mathrm{~ns} \\
\text { spacing }\end{array}$ \\
\hline 0.9 & 0.55 & 0.40 & 0.06 & 0.05 & 0.01 & 0.008 \\
\hline 1.0 & 0.60 & 0.50 & 0.08 & 0.07 & 0.014 & 0.012 \\
\hline 1.1 & 0.70 & 0.55 & 0.1 & 0.085 & 0.018 & 0.016 \\
\hline 1.2 & 0.90 & 0.75 & 0.145 & 0.150 & 0.026 & 0.025 \\
\hline 1.3 & 1.3 & 0.9 & 0.24 & 0.370 & 0.05 & 0.075 \\
\hline 1.4 & 1.4 & 1.1 & 0.76 & 1.14 & 0.18 & $>1.0$ \\
\hline
\end{tabular}

(a)
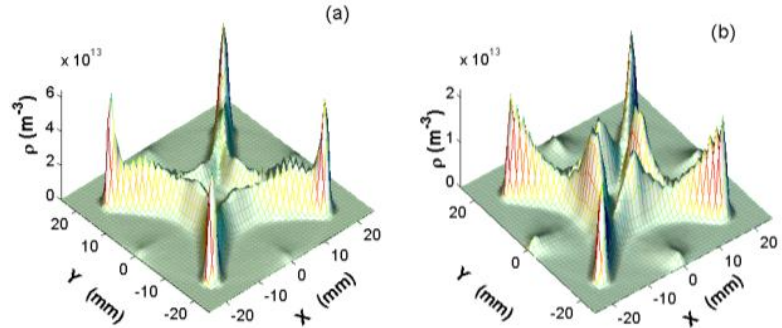

FIG. 10. Electron cloud distribution in ILC quadrupole, parameters used in simulation: $3 \mathrm{~km}$ ring, $6 \mathrm{~ns}$ bunch spacing with $98 \%$ ante-chamber reduction effect. (a) at the end of the bunch train (b) survived(trapped) electrons from the last bunch train.
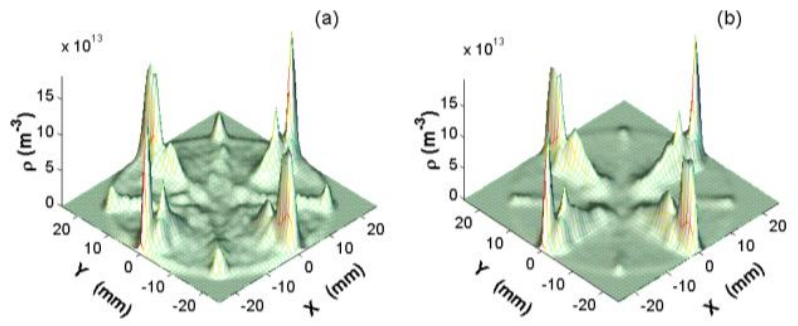

FIG. 11. Electron cloud distribution in ILC quadrupole, parameters used: $3 \mathrm{~km}$ ring, $6 \mathrm{~ns}$ bunch spacing without ante-chamber reduction effect. (a) at the end of the bunch train (b) survived(trapped) electrons from the last bunch train.

\section{SUMMARY}

We find large number of (up to 70\%) deep trapped electrons in quadrupole and sextupole magnet of CesrTA and ILC positron damping ring. Simulations show that the electron cloud in a quadrupole magnet can be deeply trapped by the mirror field. The simulation results agree with the theory well. The trapped electrons can survive the long train gaps gap of $1.93 \mu \mathrm{s}$. The distribution of trapped electrons strongly depends on the field and beam parameters. We found more electrons can be confined near the beam with a stronger magnetic field gradient. Due to the trapping, electrons in quadrupole and sextupole magnet, there is a slow decay of the electron density during the train gap and the build-up is slow. It takes up to more than 10 bunch train/turns to reach saturation level and the saturated density near the beam can be large. Theretofore, the long lifetime electron in quadrupole can be important for the beam dynamics. Moreover, CESRTA TA has strong magnetic field gradient which causes the trapped electrons closer to the beam. This may explain the observation of the effect of the long life time electron in CESRTA[6].

We systematically study the effect of bunch spacing, SEYs and ante-chamber effect for ILC damping ring. There is lower density for $6 n s$ bunch spacing and antechamber is effective on reduction of the electron density only when SEY is small $(<1.1)$. The secondary electron is dominant with a large SEY. Another important finding is that the strong space charge can significantly change the distribution of the electron cloud. It reduces the electron density near the beam. This makes the ante-chamber less attractive when SEY is large. In a summary, the quadrupole and sextupole need to be coated to reduce the SEYs in order to take advantage of the reduction of the photon flux.

\section{ACKNOWLEDGMENTS}

We thank J. Calvey, G. Dugan, B. Macek and M. Palmer for providing the input data of CESRTA and fruitful discussions. 


\section{REFERENCES}

[1]M. Palmer, et al. IPAC2010 proceedings.

[2] D. Jackson, Classical Electrodynamics.

[3] L. Wang, H. Fukuma, S. Kurokawa, K. Oide, Physical Review E, 66, 036502(2002).

[4] Joseph Calvey's talk, in this proceedings.

[5] B. Macek, in this proceedings

[6] Gerry Dugan's talk, in this proceedings. 